\title{
Fundamentos teóricos de la educación bilingüe \\ $M^{2}$ José Molina García
}

Profesora de la Facultad de Educación y Humanidades de Melilla

Univesidad de Granada. Campus de Melilla

\section{Concepto y tipos de bilingüismo}

El bilingüismo es, en la actualidad, un fenómeno social cada vez más extendido, debido a multitud de causas, entre ellas, la heterogénea procedencia de la población que forman nuestras urbes.

En su origen, según Appel y Muysken (1996), este bilingüismo nace de un contacto de lenguas frecuente, asiduo. Por ello deberíamos distinguir entre un bilingüismo social y un bilingüismo individual. El primero se produciría en sociedades en las que se habla dos lenguas o más en distinto grado y forma, lo cual nos conduce a la conclusión de que todas las sociedades, hoy por hoy, son bilingües, como es un claro ejemplo Melilla; el bilingüismo individual, por su parte, se produciría sólo en aquellos individuos que, ocasionalmente, hablan dos idiomas (por ejemplo, con motivo de un viaje a un país extranjero).

Estos autores establecen cinco posibles situaciones en las que hallaríamos contacto lingüístico:

1. Permanencia de varias lenguas en la misma ecosfera, lo que denominaríamos archipielago lingüistico. Hoy son poco habituales, pero sí fueron muy frecuentes en la época precolonial.

2. En fronteras más o menos estables entre familias lingüísticas (como es el caso de Melilla).

3. En situaciones de expansión colonial.

4. En bolsas individuales de hablantes de lenguas minoritarias aisladas por las lenguas nacionales más próximas (como en el caso del euskera en España).

5. Cuando se produce un movimiento migratorio pero de sentido inverso. 
Pero, ¿̇qué es un bilingüe? ¿cuál es la definición del ideal de bilingüe? Mesa Franco y Sánchez Fernández (1996) recogen la definición de Siguán (1986): "Persona que, además de su primera lengua, tiene competencia parecida en otra y es capaz de usar una u otra según la circunstancia"; y Ruiz Bikandi (2000) la completa: "bilingüe es la persona que añade cualquier grado de conocimiento de una nueva lengua al dominio espontáneo que posee en su propia lengua".

¿Qué implicaciones se derivan de estas definiciones? Estos autores exponen que puede deducirse una serie de rasgos imprescindibles en el bilingüe ideal, tales como que:

1. Debe mantener una independencia de los dos códigos, es decir, es capaz de separar ambos, de forma que cuando emplea la lengua $X$ o la lengua $Y$, lo hace espontáneamente, sin elegir conscientemente entre las posibilidades de un código u otro, pues, de lo contrario, habría préstamos e interferencias (que explicaremos más adelante).

2. Es capaz de alternar códigos, o sea, pasa de uno a otro rápidamente, sin excesivo esfuerzo (incluso dentro del mismo período oracional).

3. Está capacitado para traducir de un sistema lingüístico a otro, es decir, es capaz de expresar los mismos significados en los distintos sistemas lingüísticos en que es competente $o$ de transmitir un conjunto de significados comprendidos a través de la lengua $X$ mediante la lengua $Y$.

Sin embargo, ser bilingüe no es una cuestión tan simple y fácil como a primera vista puede parecer, ya que existen muchas objeciones al respecto:

1. Para empezar, es necesario ser homogéneamente competente en las, tradicionalmente llamadas, habilidades básicas, esto es, hablar, escuchar, leer y escribir, a las que Baker (1997), añade pensar. Y esto, en la práctica, resulta muy difícil, pues, en general, en unas habilidades se es más competente que en otras.

2. Son imprescindibles la fluidez conversatoria y la competencia lingüística escolar en todos los contextos y situaciones, ya que no es válido tener fluidez conversatoria en un momento y situación determinados para mantener una conversación simple (por ejemplo en una tienda en una operación de compra-venta) y no tenerla en otros contextos.

3. Se precisa lo que denomina Appel (1996) una especialización funcional o, en palabras de Baker (1997) un bilingüismo funcional, basado en las seis funciones del lenguaje, enunciadas por Jakobson, en las que se manifiesten los usos o empleos de una lengua u otra, de un código u otro, en función de la elección o competencia del hablante bilingüe (función referencial, directiva, expresiva, fáti-

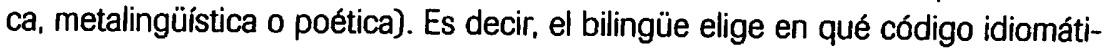
co desea realizar sus comunicaciones en medios oficiales, en sus saludos 0 modos convencionales de dirigirse a las distintas personas de su entorno, a la hora de expresar sus sentimientos, de mantener abierto un canal de comunicación en un momento determinado, de reflexionar sobre la propia lengua o de jugar con el lenguaje mediante bromas, chistes, etc.. 
En la bibliografía especifica sobre el tema, podemos encontrar muchas clasificaciones o tipos de bilingüismo, sin embargo, vamos a exponer en estas líneas la recogida por Mesa Franco y Sánchez Fernández (1996) de Sánchez y Forteza (1987) por su claridad. Esta clasificación está basada en criterios:

a. Según la relación existente entre lenguaje y pensamiento, hablaríamos de:

a.1. Bilingüismo compuesto: en el que una palabra con un significado debe pasar por una traducción para poder ser expresada en otro idioma. El aprendizaje 0 adquisición de estos códigos se hace en contextos iguales.

a.2. Bilingüismo coordinado: en el que no se precisa la traducción, por lo que podríamos decir que se trata de dos sistemas lingüísticos independientes. EI aprendizaje de códigos se realiza en contextos distintos.

a.3. Bilingüismo subordinado: en el que es necesaria una sustitución fonológica en un sistema por otro. Este tipo de bilingüismo es incluido por muchos autores dentro del anterior tipo.

b. Según la competencia alcanzada en cada uno de los idiomas, asistiríamos a un:

b.1. Bilingüismo equilibrado: en el que el usuario tiene una competencia similar en ambas lenguas.

b.2. Bilingüismo dominante: en el que se domina mejor un idioma que otro.

c. Según la edad de adquisición de las lenguas conocidas, distinguiríamos entre:

c.1. Bilingüismo precoz o temprano: que se da en la primera infancia y en el que se incluye tanto el adquirido en el seno familiar, como aquel que se produce cuando el niño accede, con una lengua materna $X$, a una escolarización temprana en el que la lengua de comunicación es $\mathrm{Y}$, con la consiguiente separación de funciones debidas a esta situación social determinada.

A la vez, este bilingüismo puede subdividirse en bilingüismo simultáneo (si la adquisición de ambas lenguas se produce a la vez), y bilingüismo sucesivo (si un idioma se adquiere antes que el otro $o$ a una edad y a otra).

c.2. Bilingüismo tardío.

d. Según el prestigio sociocultural de las lenguas, hablamos de un:

d.1. Bilingüismo aditivo: en el que se produce una valoración similar de ambas lenguas (algunos autores lo denominan bilingüismo armonioso).

d.2. Bilingüismo sustractivo: en el que la valoración de la segunda lengua se produce a expensas de la primera. Este bilingüismo nos lleva a la distinción entre bilingüismo y diglosia del que hablaremos un poco más adelante.

e. Según la identidad cultural, presenciaríamos un:

e.1. Bilingüismo bicultural: en el que en el individuo existe una doble pertenencia y. por tanto, una doble identidad cultural.

e.2. Bilingüismo monocultural: en el que se produce la identidad cultural sólo en una lengua. 
e.3. Bilingüismo acultural con anomia (sin nombre): en el que el individuo no se identifica con ninguna lengua, bien por no haber una identidad cultural, bien porque ésta esté mal definida.

De todo esto se deduce la enorme complejidad del fenómeno que tratamos.

Si es difícil hallar bilingües equilibrados, tampoco será lógico definir sociedades equilibradas desde el punto de vista del bilingüismo. Actualmente, es casi imposible encontrar una ciudad en la que no habiten personas de distinta procedencia, incluso con distintas lenguas. Estas lenguas que cohabitan en un territorio están adscritas a diferentes estatus sociales y culturales y se produce, en general, entre ellas, un desequilibrio a favor de una (por relaciones sociales, económicas, políticas, etc), lo cual implica clasificarlas de "lengua fuerte"/ "lengua débil" o "lengua mayoritaria"/ "lengua minoritaria", etc. Es lo que denominamos con el término de diglosia.

Autores como Baker (1997) o Siguán (1986), puntualizan que, en la práctica, es dificil que una comunidad lingüística use dos lenguas para el mismo propósito, si bien, al contrario, una es utilizada para distintas situaciones y con distintas funciones que la otra. Además, añaden que la lengua fuerte suele tener funciones superiores en la vida social, se utiliza en los niveles sociales altos y en la ciudad y dispone de vocabulario para todos los campos cientíicos; mientras que, la lengua débil, es más familiar, se trata de una lengua oral y, por tanto, no normativizada, se utiliza en niveles inferiores y en zonas rurales y no siempre dispone de vocabulario específico en cualquier campo. En el caso de nuestra ciudad, esta distinción de estatus se cumple entre el castellano y el amazige, ya que, esta última lengua no está codificada (muchos de sus hablantes no saben escribirla) y se transmite de generación en generación por vía oral, por lo que es muy vital y existe de forma independiente al árabe, aunque utiliza y adopta palabras tanto de este idioma como del castellano, a veces por falta de vocabulario específica.

Es obvio que la mayor o menor vitalidad de una lengua viene dada por una serie de factores (recogidos por Baker (1997), de estatus (económicos, sociales, simbólicos), demográficos (distribución geográfica, matrimonios mixtos interlingüísticos), de apoyo institucional (MAV, servicios administrativos, mass media), que es necesario considerar. Por todo ello, podemos resumir diciendo que el bilingüismo es objeto de estudio de psicólogos y lingüistas y es estudiado desde un punto de vista individual como la capacidad de ese individuo de utilizar más de una lengua. Mientras que la diglosia es objeto de estudio de socí́logos y sociolingüistas y es estudiado desde un punto de vista social para averiguar e investigar sobre el estatus social de las lenguas.

En Melilla, la realidad es que a nuestras escuelas acuden niños, cuya lengua materna es el amazige y se da el caso de que, en algunas ocasiones, sí utilizan el código castellano (aunque con algunas carencias) pero en atros no. Además, tanto en un caso como en otro, padecen dificultades de comunicación e infrarendimiento (por deficiencias en asociación, comprensión e integración auditivas, integración gramatical, comprensión y expresión verbales, asociación, compresión e integración visuales, nivel viso-motor] por lo que es preciso tomar medidas y planificar estrategias. 
A este respecto, en el terreno educativo, la UNESCO defiende la utilización o referencia en la escuela de las lenguas minoritarias, según recogen Appel y Muysken (1996) exponiendo los siguientes argumentos:

1. Para que el progreso académico del alumno no se vea afectado.

2. Para evitar un retraso en el desarrollo cognitivo (en relación con la llamada hipótesis del umbral).

3. Resulta imprescindible para la personalidad del niño.

4. Suaviza el impacto cultural que se produce en el acceso a la etapa educativa de estos niños.

5. La influencia que el desarrollo de la lengua materna puede tener en el desarrollo de la lengua de adquisición (hipótesis del desarrollo independiente).

6. Para evitar la forzada asimilación lingüística y cultural de los grupos minoritarios gracias a la enseñanza de la lengua minoritaria.

7. Para mejorar la relación social merced al reconocimiento en la escuela de la lengua minoritaria.

Respecto a los modelos educativos que podemos aplicar y que estudiaremos más adelante, hemos de decir que no siempre son totalmente transferibles porque cada país del que son origen, posee una características específicas, una metodología particular (nunca totalmente idéntica) y un contexto educativo, social,... determinados. Aún así podemos tomarlos como referencia o punto de partida y diseñar los propios. Baker (1997) recoge los de Fischman que los clasifica en compensatorios, de mantenimiento y de enriquecimiento. Por otro lado, Appel y Muysken (1996) hablan de modelos de transición o asimilacionista, pluralista o de mantenimiento o de protección lingüística, de inmersión y de sumersión.

Para poder valorar cuál es la situación inicial con la que nos enfrentamos y desde la que partimos, es aconsejable recurrir a las escalas de origen lingüístico o a los tests de medidas de equilibrio lingüístico (Baker, 1997).

En las primeras podemos recopilar información sobre los contextos y situaciones en las que los individuos utilizan uno u otro código lingüístico, asi como las funciones del lenguaje utilizadas con cada uno de ellos, mediante preguntas como "¿en qué lengua hablas tú a las siguientes personas?", "¿en qué lengua te hablan estas personas?", “¿qué lengua utilizas tú en los siguientes casos?" o "¿con qué frecuencia utilizas esta lengua u otra?" Incluso podría acompañarse de una autoevaluación, según la edad de nuestros alumnos.

En las segundas, se trata de medir el equilibrio o dominancia entre las dos lenguas. mediante pruebas de velocidad de reacción en una tarea de asociación de palabras, cantidad de reacciones a una tarea de asociación de palabras, detección de determinadas palabras según un criterio, tiempo empleado en leer un texto o la cantidad de interferencias o mezclas que se producen en las conversaciones propuestas,...

Aunque si bien, todo esto sólo mide una pequeña parte de subdestrezas lingüísticas, deben proporcionarnos, según Baker (1997) otras informaciones como:

1. Decisiones sobre qué enseñar.

2. Qué información dar a los padres, ya que el apoyo familiar es imprescindible. 
3. Detectar a los niños que necesiten un apoyo especial.

4. Separar a los niños que necesiten un aprendizaje más acelerado.

5. Qué niveles se han alcanzado o puedan alcanzarse en clase...

Es importante establecer metas lingüísticas, basadas en el enfoque comunicativo, para fijar unos objetivos a alcanzar y unas tareas de evaluación por las que valoremos los resultados que se van obteniendo, todo ello adecuado y adaptado a las edades de los alumnos con los que trabajemos, por ejemplo, Baker (1997) propone que, aproximadamente, a los cinco años una meta lingüística podría ser participar como hablante y oyente en actividades de grupo, como por ejemplo, representar roles en un juego simbólico e imaginativo y la tarea de evaluación sería plantear una situación como la de "jugar a las tiendas" donde un niño es el tendero y otro el cliente; o a los nueve años. una meta establecida sería dar, recibir y seguir con precisión instrucciones mientras realiza una tarea como individuo o como miembro de un grupo y la evaluación podría ser planificar un mural y organizar una excursión en grupo.

\section{Adquisición y aprendizaje de una segunda lengua}

Todo lo expuesto en el apartado anterior nos conduce, inevitablemente, a reconocer una cuestión que parece ser de dominio público entre los profesionales que nos dedicamos a la educación y es que el factor edad es fundamental a la hora de aprender una segunda lengua. En los años setenta, cuando los estudios se centraban sobre los mecanismos que llevaban al niño al aprendizaje de su lengua materna, surge la necesidad de distinguir entre los términos adquisición y aprendizaje de una lengua. Y se concluye que la adquisición es un proceso consciente e intuitivo que no precisa una instrucción (como es el caso de los bebés cuando, antes de acceder al sistema educativo, ya conocen su lengua y la utilizan con cierta soltura), mientras que, en el caso del aprendizaje, si se trata de un proceso consciente en el que se precisa algún grado (según el caso) de instrucción.

Por otro lado, y según Ellis (1985) en el desarrollo de una segunda lengua, existen tres partes 0 aspectos claramente diferenciados: la secuencia en el aprendizaje (son estadios generales por los que se pasa a medida que se aprende el idioma, por ejemplo desde el aprendizaje de un vocabulario simple a la formación de la oración compleja, es imprescindible dominar antes una sintaxis básica y las estructuras oracionales simples); orden de esos conocimientos gramaticales o lexicales que varían según las características de la persona, la situación, etc.; y una tasa o medida de desarrollo de esa segunda lengua y el nivel de competencia logrado, en el que también influyen los factores de situación, actitud, motivación, estrategias de aprendizaje puestas en práctica y personalidad. Todos estos pasos se hacen más conscientemente conforme se avanza la edad, por lo que nos lleva a deducir que, cuanto más tempranamente adquiera la persona la segunda lengua, más se aproximará a los procesos de adquisición que de aprendizaje.

Efectivamente, en el bilingüismo temprano simultáneo, que vimos antes, los padres se comunican con el bebé en distintos códigos y en el secuencial el niño asistirá a la comunicación en dos lenguas, una en casa y otra en la escuela. Sin embargo, aunque hasta los 
dos años, aproximadamente, mezclará códigos, poco a poco aprenderá a separarlos y aprenderá a dirigirse y a seleccionar la lengua de comunicación en una situación o en otra, de forma casi espontánea. Este proceso pierde su naturalidad con la edad, es decir, si el bilingüismo es tardío. En nuestras aulas, incluso en las de Educación Infantil, muchos niños han aprendido a utilizar un idioma (el castellano) y otro en casa (el amazige).

¿Quiere esto decir que no es posible el éxito o la eficacia en el bilingüismo tardío? No, rotundamente, no. Sólo que el proceso será menos intuitivo y entrará en juego otra serie de factores.

En los niños (hasta los ocho años), el momento de adquisición o aprendizaje es óptimo, pues los mecanismos corren paralelos a los de la adquisición de su propia lengua materna y el éxito o eficacia es el cien por cien. En los adolescentes (hasta los catorce años], los mecanismos de aprendizaje no guardan similitud con los que pusieron en marcha en su lengua materna y, aunque su ansia de comunicación es muy grande, lo cual le lleva a cometer muchos errores y a pasar por fases de interlengua (que luego definiremos), con una buena guía orientativa concluirán su aprendizaje con éxito. Sin embargo, en el caso de los adultos (desde los catorce años en adelante), influyen otros factores psicológicos, culturales, de aplicación de estrategias propias de aprendizaje, de apego a la gramática, etc, que hacen el éxito muy variable, pues se producen estancamientos y fosilizaciones en las interlenguas y el aprendizaje ya no es, en absoluto, intuitivo por lo que precisa, de orientación profesional (Villalba Martínez et alt., 1999). Las fases de interlengua a las que antes aludíamos en los casos de los adolescentes y adultos, no son más que las versiones o variedades que se producen en la lengua de llegada del individuo a medida que aprende una segunda lengua (Selinker, 1972). Estas versiones o fases son perfectamente predecibles y los errores que en ellas se cometen, forman parte del proceso de maduración en el aprendizaje de esa segunda lengua. Podíamos compararlas con las expresiones y construcciones que los bebés manifiestan cuando descubren el lenguaje humano, y que, a menudo, provocan la risa de los familiares de su entorno; son errores que forman parte de un proceso de maduración, hasta llegar a la expresión correcta y que debemos ayudar a superar para evitar la fosilización de construcciones agramaticales o inadmisibles en el idioma.

No deben confundirse estas fases sucesivas de interlenguas con las interferencias lingüísticas, pues éstas últimas son errores que se producen al querer aplicar estructuras de la lengua materna a la segunda lengua a pesar de no ser transferibles.

Lo que sí es cierto es que la lengua materna o L1 influye, inevitablemente [ya hemos visto que en adolescentes y adultos más] en la $L 2$, pero también en la interlengua, pues el tiempo de aprendizaje invertido guarda una relación de proporción directa a la semejanza que mantengan entre sí las construcciones entre la L1 y la L2, es decir, si una construcción sintáctica determinada en L2 es muy distinta a la que el individuo conoce de su L1, supondrá para él un mayor esfuerzo de asimilación.

Por otra parte, las fases de la interlengua pueden variar, dependiendo de factores y circunstancias diversas o pueden fosilizarse (por ejemplo si el individuo no quiere superar errores porque, aunque sea consciente de ellos, no le impiden la comunicación) o 
puede producirse una regresión involuntaria (cuando se sienten inseguros ante la aplicación de nuevas construcciones y retroceden a etapas anteriores que ya habían sido superadas) e incluso pueden transferir construcciones de la $L 1$ a la interlengua por procesos de generalización o simplificación de reglas, etc.

Otro fenómeno, también aludido antes, al que podemos asistir es el de la alternancia o mezcla de códigos, a modo de coletillas, en el interior de las frases o alternancia de frases en ambos idiomas. No es extraño escuchar, en una conversación entre individuos de origen bereber, esta alternancia y también podemos encontrarla en nuestras aulas con nuestros alumnos, es decir, que en una misma conversación alternen expresiones o palabras en amazige y en castellano. Será función del educador tratar de subsanar estas interferencias y mezclas, así como orientar para superar las fases de interlengua. Sin embargo, no podemos confundir estas interferencias y mezclas de códigos con la utilización de préstamos léxicos, ya que éstos son también muy usuales en las conversaciones entre individuos de habla amazige, debido a la carencia de vocabulario específico que antes mencionábamos, o la voluntad de utilizar sinónimos más expresivos, o para evitar la ambigüedad, o, simplemente, por el hábito inconsciente de ser bilingües de dos lenguas en constante contacto.

Es inevitable hacer una referencia, aunque sea breve, a las teorias de adquisición de la primera lengua, porque influirán también en la orientación de los profesionales a la hora de planificar su actuación educativa. Según si tenemos una visión conductista, innatista, computacionalista, constructivista, socio-cultural o interaccionista, la praxis docente seguirá una metodología u otra.

Respecto a las teorías de adquisición de la segunda lengua, se tiñen todas ellas de rasgos psicológicos y supuestos sociológicos (no nos detendremos en explicarlas por falta de espacio y tiempo), enfatizando aquellos elementos en los que se basan los mecanismos de adquisición y procesamiento de información. En lo que sí están todas conforme es en los factores que influyen en esta adquisición de la segunda lengua, o sea: la priorización de la práctica por encima de la teoría (en actos de comunicación dados en pequeñas dosis, con un número de alumnos determinado), en la competencia lingüística del discente y en sus características personales (edad, aptitud, motivación, actitudes, personalidad, uso de estrategias].

Par ende, todos aquellos supuestos que rigen la potenciación de la lengua oral en el aula, son totalmente válidos, esto es: intercambios lingüísticos de calidad y en cantidad, participación del alumno en la selección de estos actos comunicativos asegurándonos así la motivación y el interés, la variedad de situaciones y contextos y, icómo no! que el alumno se sienta libre y cómodo para preguntar y participar en su propio aprendizaje.

\section{Las segundas lenguas y la escuela}

Es obvio que, para acometer la tarea de la planificación lingüistica en la escuela, se requiere unos supuestos teóricos. También es cierto que hay aspectos que sólo corresponden a una nación, como delimitar qué lengua será la nacional, cómo tratar las lenguas minoritarias (si serán permitidas, promovidas o usadas y aceptadas en los ámbitos administrati- 
vos], así como el desarrollo lingüístico que se les confiere. Pero, al margen o paralelamente a todo esto, el Centro Educativo tiene la flexibilidad, otorgada por la LOGSE, de establecer un proyecto lingüistico en el centro educativo cuando su comunidad escolar lo precise por la presencia de alumnos de lenguas minoritarias, en el que se diriman decisiones tan importantes como el momento de introducción de cada una de las lenguas, su grado de presencia en el currículum y la concepción instrumental de la misma, el tratamiento a lo largo del programa, la progresión y proporcionalidad en el tiempo de instrucción de las lenguas durante las distintas etapas educativas y la tipología lingüística del aula como uno de los factores clave en el programa, asi como el papel reservado a la familia (Baker, 1997). De este modo, se podrán subsanar algunas causas de infrarendimiento escolar en alumnos de lengua materna minoritaria por la falta de exposición a la lengua mayoritaria, el desajuste entre el hogar y la escuela, el tipo de escuela al que asisten así como aclarar si ese infrarendimiento se debe a factores socio-económicos (que interactúan con los demás) o si hay dificultades reales de aprendizaje que se agravan con el bilingüismo.

Los distintos paises con tradición en enseñanza bilingüe, han diseñado modelos muy variados, recogidos y caracterizados por Baker (1997). Así distinguiríamos, muy brevemente:

1. Enseñanza por sumersión: en el que se obvia totalmente la lengua materna del alumno.

2. Enseñanza con clases de retirada, esto es, con clases de compensación en la L2.

3. Enseñanza segregacionista, en las que se contempla dar clases en L1.

4. Enseñanza bilingüe transitoria: en donde se les enseña lo justo para comunicarse y puedan emplear la L1. El trabajo de conversión lo realiza, por lo general, un bilingüe.

5. Enseñanza general, muy relacionada con la enseñanza de una lengua extranjera, en la que los niños reciben clases en L1 y una clase adicional en $L 2$, con lo que esta lengua se convierte en una asignatura más. Este tipo de enseñanza no hace verdaderos bilingües.

6. Enseñanza separatista: promovida por la propia comunidad para que su lengua materna no sea arrollada por una L2. Este tipo de enseñanza es poco usual en la escuela oficial.

7. Enseñanza por inmersión: en la que se tiene en cuenta factores de edad, tiempo, etc y se marca como objetivo hacer verdaderos niños bilingües.

8. Enseñanza de mantenimiento y enseñanza bilingüe en lengua patrimonial: en las que se emplea esta lengua en la escuela como medio de instrucción y cuya meta es el bilingüismo.

9. Enseñanza bilingüe de doble dirección en dos lenguas: cuyas aulas se llenan con niños de distintos idiomas (a idéntica proporción) y en las que se establecen horarios de clases alternas (por ejemplo, los lunes, miércales y viernes en $L 1$ y los martes y jueves en L2). Además se cuenta con la familia para aportar y explicar aspectos culturales.

10. Enseñanza bilingüe generat: en el que se contemplan ambas lenguas a la vez en el plan de estudios. Este tipo de enseñanza se suele dar en los países europeos donde se reconocen como oficiales ambas lenguas, por ejemplo en Luxemburgo [luxemburgués, francés o alemán]. 
Ruiz Bikandi (2000) complementa esta visión optando por el programa de inmersión lingüistica como uno de los de más probada eficacia, ya que se basa en los siguientes presupuestos psicopedagógicos:

a. Están dirigidos a alumnos de lengua y cultura mayoritaria.

b. Se parte de un desconocimiento inicial de la lengua que se habla en la escuela (L2) por parte de los alumnos.

c. Se basan en una concepción instrumental de la enseñanza de la lengua.

d. Se solicita la participación voluntaria de los padres.

e. El profesorado es bilingüe.

f. Los maestros enfatizan los aspectos comprensivos de la lengua a la vez que crean contextos de uso que permiten a sus alumnos el dominio gradual de una segunda lengua.

g. Utilizan una metodología acorde y adecuada a estos presupuestos.

En esta visión pedagógica de métodos y líneas que hemos expuesto pueden enmarcarse algunos de los modelos de programas bilingües que se siguen en España. Así, por ejemplo en el País Vasco y Navarra, el euskera puede ser tratado como una lengua extranjera, puede monopolizar toda la enseñanza recibida como única lengua de comunicación o puede ser utilizada como lengua instrumental en algunas asignaturas y reservar el castellano para la lectura, escritura y matemáticas, en igual número de horas; en el País Valenciano, los niños reciben en la escuela una primera etapa intensiva (hasta los cinco años] en valenciano para luego ir introduciendo paulatinamente el castellano como lengua vehicular; en Cataluña, pionera en programas bilingües, este modelo de inmersión está más desarrollado y es más completo, dotando de un tratamiento instrumental a ambas lenguas (castellano y catalán), priorizando el lenguaje oral sobre el escrito y dotando a estos idiomas de un porcentaje presencial que va progresivamente aumentando conforme se avanza en los cursos, a favor del castellano, aunque el catalán sea el más utilizado. A esto se añade una lengua extranjera (asi en El-2 ${ }^{\circ}$ de EP el porcentaje es de catalán un $90 \%$ y castellano un $10 \%$; en $3^{\circ}-4^{\circ}$ de EP el catalán es un $70 \%$, el castellano un $20 \%$ y la lengua extranjera un $10 \%$; y en $5^{\circ}-6^{\circ}$ de EP la instrucción en catalán es de un $60 \%$, en castellano de un $30 \%$ y en lengua extranjera se mantiene en el $10 \%$ ).

En el caso concreto de Melilla, no existen programas de este tipo (por falta de medios, recursos y, quizá, infraestructura), pero sí hay una inquietud ante la situación de nuestras aulas, por parte del profesorado por paliar las causas del infrarendimiento que antes mencionábamos y ofrecer ayuda a los alumnos que lo precisan.

A tal efecto, vamos a comentar, aunque sea sucintamente, la intervención educativa llevada por Mesa Franco y Sánchez Fernández (1996) en distintos centros de nuestra ciudad, representativos de tres clases sociales distintas: C.P. "Reyes Católicos", C.P. "Velázquez" y C.P. "León Solá", representativos de una clase media-alta, media-baja y baja, respectivamente. El estudio de campo y la intervención educativa se realizaron desde El hasta el tercer curso de EGB.

Estos autores, al detectar todas las deficiencias y carencias que antes mencionábamos, (136) decidieron realizar una práctica educativa que, si bien no tenía como fin hacer verda- 
deros bilingües, si contribuiría a subsanar gran parte de estas deficiencias y carencias. Cuatro son los supuestos básicos que se trazaron en su trabajo:

1. Respetar la secuencia habitual de actividades didácticas.

2. Trabajar muchas actividades específicas de lenguaje, sobre todo con cuentos, previa labor rigurosa de selección.

3. Diseñar juegos y actividades para el desarrollo didáctico del lenguaje, sobre todo de vocabulario.

4. Planificar estrategias de apoyo y motivación familiar, ya que, muchas veces la cerrazón de la familia supone un obstáculo en la enseñanza bilingüe.

Con todo ello consiguieron importantes mejoras en comprensión auditiva, asociación auditiva, expresión verbal, integración gramatical, etc y también en la estimulación familiar.

No debemos pensar que el bilingüismo es, pues, una amenaza, algo que se nos viene encima, algo nefasto. Si bien, al contrario, es necesario verlo como un enriquecimiento individual y social. A tal efecto, se ha estudiado y demostrado los posibles efectos del bilingüismo sobre la persona. Exponemos aquí algunas de las conclusiones a las que se ha llegado (Baker, 1997):

1. Bilingüismo y cerebro: Realmente, no está demostrada esta relación pero lo que sí es cierto es que no supone una carga extra para el individuo. Si nos basamos en el hecho probado de que el Hemisferio Izquierdo del cerebro se ocupa de los aspectos más analíticos del lenguaje (por ejemplo de la sintaxis) y el Hemisferio Derecho de aspectos más pragmáticos y emotivos, en los bilingües, sobre todo en los tardios, la utilización de estos últimos aspectos es mayor que en los monolingües.

2. Bilingüismo y pensamiento: En general, se relaciona al bilingüe con un pensamiento divergente y creativo, es decir, con una mayor fluidez, flexibilidad, originalidad y elaboración.

3. Bilingüismo y conciencia metalingüística: Es un hecho probado que para el bilingüe, la relación entre la palabra y el significado es más débil. Son flexibles y se adaptan más. También son superiores en los procesos lingüísticos de control cognitivo y están más capacitados para detectar errores y corregirlos.

4. Bilingüismo y sensibilidad comunicativa: Como cambian constantemente de lengua según la situación, contexto, función, etc, son más sensibles y conscientes de las necesidades del oyente.

Ya vemos que, en el terreno educativo, aún quedan muchas cosas que hacer en el tema del bilingüismo. Sin embargo, mientras los profesionales del sector estén interesados y muestren una actitud receptora y de toma de iniciativas, estaremos en el buen camino, ya que lo importante es analizar la situación de nuestras aulas y manifestar voluntad por subsanar los obstáculos que en ella se planteen. En foros del tipo de este $\checkmark$ Curso de Multicultura al que asistimos, podemos reflexionar sobre el tema e intentar dar soluciones al respecto, ante una realidad que no se avecina, sino que convivimos diariamente con ella. 


\section{Referencias bibliográficas} sobre bilingüismo

- APPEL, R. y MUYSKEN, P. (1996), Bilingüismo y Contacto de Lenguas, Barcelona. Ariel.

- BAKER. C. (1997), Fundamentos de Educación Bilingüe y Bilingüismo, Madrid, Cátedra.

- ELLIS, R., (1985], Understanding Second Language Acquisition, Oxford, Oxford University Ress.

- MESA FRANCO, M. C. y SÁNCHEZ FERNÁNDEZ, S. (1996), Educación y situaciones bilingües en contextos multiculturales. Estudio de un caso: Melilla, Madrid, CIDE.

- RUIZ BIKANDI, U. (Ed.) (2000), Didáctica de la segunda lengua en educación infantil y primaria, Madrid, Sintesis.
- SANCHEZ, M.P. Y FORTEZA. J. A., (1987), Bilingüismo e inteligencia.En YELA, M., (Dir.): Estudios sobre inteligencia y lenguaje, Madrid. Pirámide, pp. 201-254.

- SELINKER, L., (1972), Interlanguage en International Review of Applied Linguistics, 10, pp. 209-231.

- SIGUÁN, M. y MACKEY, W. F., (1986). Educación y bilingüismo. Madrid. Santillana-Unesco.

- VILLALBA MARTÍNEZ, F., HERNÁNDEZ GARCÍA, M. T. Y AGUIRRE MARTÍNEZ, C., (1999), Orientaciones para la enseñanza del español a inmigrantes y refugiados, Madrid, MEC. 